\title{
Trophoblast replacement enables rhesus monkey cloning by somatic cell nuclear transfer
}

Qiang Sun ( $\nabla$ qsun@ion.ac.cn )

Institute of Neuroscience

Brief Communication

Keywords:

Posted Date: March 1st, 2022

DOI: https://doi.org/10.21203/rs.3.rs-1371807/v1

License: (c) (i) This work is licensed under a Creative Commons Attribution 4.0 International License. Read Full License 


\section{Abstract}

Somatic cell nuclear transfer (SCNT) has been used to clone cynomolgus monkeys, but cloning of other non-human primate species remains to be achieved. Our histological examination indicated severe calcification of the placenta of implanted SCNT macaque embryos. By replacing SCNT-derived trophoblast with ICSI-derived trophoblast, endowing a normal placenta for the SCNT fetus, we obtained a healthy SCNT rhesus monkey. Thus, trophoblast replacement represents a useful approach for rhesus monkeys cloning.

\section{Main}

Somatic cell nuclear transfer (SCNT) technology has been widely applied in cloning mammalian species, including cattle ${ }^{1}$, swine ${ }^{2}$, goat ${ }^{3}$, rabbit ${ }^{4}$ and dog $^{5}$. Wild-type and gene-edited cynomolgus monkeys (Macaca fascicularis) have recently been cloned by SCNT via optimizing nuclear transfer protocols and application of epigenetic regulators, including histone demethylase $K d m 4 d$ and deacetylation inhibitor trichostatin A (TSA) 6,7. However, SCNT cloning of rhesus monkey (Macaca mulatta), an extensively used NHP species for basic and clinical research ${ }^{8-11}$, has not been achieved so far.

In this study, we first found that the protocol used for SCNT cloning of cynomolgus monkeys ${ }^{6,7}$ failed to produce healthy live birth of rhesus monkey. Furthermore, we found that SCNT monkey placentas showed severe premature calcification. We thus developed a trophoblast replacement method, in which the trophoblast of the SCNT embryo was replaced by that of the ICSI embryo. By this method, we were able to achieve live birth of a SCNT rhesus monkey, which remains healthy at the present time (20 months after birth).

Similar to the SCNT method previously reported for cloning cynomolgus monkeys, we applied a combination of epigenetic regulators $K d m 4 d$ and TSA in cloning rhesus monkey. We found that the rate of forming morphologically normal blastocysts of rhesus monkey SCNT embryos reached $47.6 \%$ (10/21), a level comparable to that found for cynomolgus SCNT cloning (44.7\% and 55.8\%, Supplementary Fig. $1 a, b)$. Over a two-year period, we have transferred in total 484 rhesus SCNT embryos into 96 surrogates ( 5 embryos per surrogate, Table 1), and obtained 22 confirmed pregnancies (carrying 33 fetuses), comparable to that of cynomolgus SCNT embryos. One live birth of SCNT rhesus monkey was obtained, but it survived for only 23 hours (Table 1 ). This rate of live birth $(0.2 \%)$ was much lower than that reported previously for cynomolgus monkey $(2.5 \%$ and $1.5 \%$, see ref. 6,7$)$ (Table 1 and Supplementary Fig. $1 \mathrm{c}$ ). Furthermore, we found all of the SCNT rhesus embryos that had specific date of abortion (31 out of 32) were aborted before gestation day 120 (Supplementary Fig. $1 \mathrm{~d}$ and Supplementary Table 1). This suggests the developmental impairment during the post-implantation period of SCNT rhesus embryos.

Abnormal placenta development has been reported in many mammalian cloning studies ${ }^{12-14}$. Our sonographic examination revealed that aborted monkey SCNT placentas during late fetal stages exhibited higher calcification than full-term ICSI and SCNT placentas (Supplementary Fig. 2). Using 
Alizarin red (AR) staining, we found that placentas from late stage-aborted SCNT fetuses indeed showed heavier dots or clouds of calcified regions (Fig. 1a). The average calcification index (CCl, percentage area showing calcification, see Methods) for placentas of the late stage-aborted SCNT placentas was $2.3 \pm 1.3$ $\%$ (s.e.m., $n=4$ placentas, 2 35 sections/placenta, Supplementary Table 2), whereas that of placentas from ICSI embryos and SCNT full-term embryos were all below 1\% (Fig. 1b). Furthermore, we also found that the average placenta thickness of late stage-aborted $(n=3)$ and full-term SCNT $(n=5)$ placentas was nearly 1.5 -fold of that found in full-term ICSI placentas ( $n=3$ ) (Fig. 1a,c and Supplementary Table 2). Thus, severe placenta abnormality may have impeded the development of SCNT fetuses.

To overcome the potential developmental impairment due to defective placenta, we developed a trophoblast replacement method. To perform SCNT cloning, we used cultured fibroblasts from the tissue of an aborted male rhesus fetus. The inner cell mass (ICM) of a SCNT blastocyst was obtained by immuno-surgical removal of its trophoblast and injected into an ICSI blastocyst, whose ICM was removed by micromanipulation immediately after introduction of SCNT-ICM (Fig. 1d, Supplementary Fig. 3 and Supplementary Movie 1, see Methods).

Using this trophoblast replacement method, we generated 11 reconstructed embryos out of 113 activated rhesus SCNT embryos, and transferred the reconstructed embryos into 7 female rhesus surrogates (Table 1 and Supplementary Table 3). Ultrasound examination showed that two surrogates became pregnant (Fig. 1e), with one carrying twins. The twins aborted on day 106 of gestation, while the singleton was born with healthy condition on day 157 (Fig. $1 \mathrm{f}$ and Table 1). Short tandem repeats analysis was performed to confirm its genomic origin. The nuclear genome of the cloned monkey was identical with the fibroblast cell line at all of the 29 loci examined (Fig. $1 \mathrm{~g}$ and Supplementary Table 4). The mitochondria origin of this cloned monkey was confirmed by the identity of single nucleotide polymorphisms (SNPS) of mitochondrial DNA with those of the oocyte donor monkey (Fig. 1h). We noted, however, the placenta of this cloned rhesus monkey comprised of cells from ICSI and SCNT embryos (Fig. $1 \mathrm{~g}$ and Supplementary Table 4), indicating that the injected SCNT-ICM contributed in part to the placenta of reconstructed embryo. Although the full-term birth rate of rhesus monkey cloning was still lower than that of cynomolgus monkey cloning, trophoblast replacement not only improved the live birth rate from $0.2 \%(1 / 484)$ to $0.9 \%(1 / 113$, Fig. $1 i)$, the implantation rate was also increased from $7.2 \%(35 / 484)$ to $27.3 \%(3 / 11$, Table 1$)$.

We compared the placentas of SCNT, ICSI and reconstructed embryos to investigate whether trophoblast replacement method had helped to prevent the placenta defects (Fig. 1a-c, Supplementary Fig. 2 and Supplementary Table 2). The CCI of SCNT, ICSI and reconstructed placentas showed that the calcification level of full-term reconstructed placenta was indeed significantly lower than that in late-stage-aborted SCNT placentas (Fig. 1b and Supplementary Table 2). Since both full-term SCNT and reconstructed fullterm placentas bear similar calcification level as full-term (Fig. 1a,b and Supplementary Fig. 2d, Supplementary Table 2), we concluded that the correction of premature calcification had improved the post-implantation development of cloned fetus, although the thickness of placenta for the reconstructed embryo remained similar to that of the SCNT embryo (Fig. 1a,c). Together, our results demonstrated that 
trophoblast replacement partially prevented the abnormal placenta phenotypes of monkey SCNT embryos and we successfully obtained the world's first cloned rhesus monkey by SCNT.

Tetraploid embryo complementation-based trophoblast replacement method also improves SCNT cloning efficiency in mice ${ }^{12}$. Here we showed that the replacement of SCNT trophoblast with normal ICSI trophoblast, in combination with the previous optimized SCNT protocol ${ }^{6,7}$, improved the full-term birth rate of SCNT cloning of rhesus monkey from $0.2 \%$ to $0.9 \%$, with one healthy clone survived for 1.5 year up to the present. Thus, trophoblast replacement may represent a useful approach in SCNT cloning of non-human primates for future biomedical research.

\section{Declarations}

\section{Acknowledgements}

We thank Mu-ming Poo for comments on the manuscript, other members of our laboratories for their contributions, and staffs of the non-human primate facility of Center for Excellence in Brain Science and Intelligence Technology for their assistance in animal care. This work was supported by grants from the National Natural Science Foundation of China Grant (31825018, 82021001 to Q.S.), the Strategic Priority Research Program of the Chinese Academy of Sciences (XDB32060100 to Q.S. and Z.L.); the Shanghai Municipal Science and Technology Major Project (2018SHZDZX05 to Q.S. and Z.L.); the Basic Frontier Scientific Research Program of Chinese Academy of Sciences From 0 to 1 original innovation project (ZDBS-LY-SM019 to Z.L.).

\section{Author contributions}

Q.S. and Z.L. conceptualized and supervised the project. Z.L., Z.D.L. and S.Y.S. designed the experiment. Z.D.L. and S.Y.S. performed experiments, analyzed data, and wrote the manuscript. Z.D.L. performed ICSI, SCNT, trophoblast replacement, primary fibroblast isolation and conducted embryo transfer, STR analysis, AR staining and embryo immunostaining. S.Y.S., X.Y.J., J.C. and Z.D.L. performed donor cell cultivation. Y.Z.L. performed $K d m 4 d$ in vitro transcription. Y.Z.L., S.Y.S. and Z.D.L. performed mtDNA genome sequencing and analyzing. Z.L., Z.D.L. and S.Y.S. integrated and interpreted the data. Q.S. and Z.L. provided conceptual advice and discussed results.

\section{Competing interests}

The authors declare no competing interests.

\section{Data and materials availability}

All data are available in the manuscript or the supplementary materials.

\section{Methods}




\section{Animal ethic statements}

Monkeys used in this research were from the non-human primate facility of Center for Excellence in Brain Science and Intelligence Technology, Shanghai, China. All animal procedures followed the guideline of Animal Use and Care Committees in the Shanghai Institute of Biological Science, Chinese Academy of Sciences, and in Institute of Neuroscience, CAS Center for Excellence in Brain Science and Intelligence Technology. The applications approved by the committees were entitled "The research of constructing macaque animal model by somatic cell nuclear transfer" (ION-2018002R01).

\section{Superovulation, oocyte collection, and nuclear donor cell preparation}

The procedures for superovulation and oocyte collection followed that previously reported ${ }^{6,7}$. Briefly, following priming with human follitropin and chorionic gonadotrophin, oocytes were aspirated into prewarmed HEPES-buffered Tyrode's lactate medium (TH3) containing heparin, and cultured in the preequilibrated 1066 medium for further in vitro maturation ${ }^{15,16}$. The preparation of primary fibroblasts as donor cells was performed as described previously ${ }^{6,7}$. In brief, the skin tissue of an aborted 62-day old rhesus fetus was cut into small pieces by sterile scissors, then then digested in DMEM containing DNase $(1 \mathrm{mg} / \mathrm{ml})$, collagenase IV $(0.5 \mathrm{mg} / \mathrm{ml}), 100 \mathrm{IU} / \mathrm{ml}$ penicillin and streptomycin, $10 \% \mathrm{FBS}, 1 \%$ non-essential amino acids and $1 \%$ glutamine at $37^{\circ} \mathrm{C}$ in $5 \% \mathrm{CO}_{2}$ for $4 \mathrm{~h}$. Next, the dissociated fibroblasts were allowed to proliferate for 10 20 h until confluency in the culture dish. The cultured fibroblasts were cryopreserved in medium containing $10 \%$ dimethylsulfoxide and $90 \%$ fetal bovine serum for later use. Thawed fibroblasts were further cultured for several days before nuclear transfer was performed.

\section{Monkey SCNT and ICSI}

The procedures for monkey SCNT and ICSI followed those previously reported ${ }^{6,7}$. Briefly, metaphase IIarrested oocytes were selected for SCNT and ICSI. For SCNT, 10 20 MII were placed into TH3 medium containing $5 \mathrm{mg} / \mathrm{ml}$ cytochalasin B (CB) in a glass-bottomed dish. The spindle-chromosome complexes of the oocytes were removed and HVJ-E virus-treated donor fibroblasts were injected into the perivitelline space of the enucleated oocytes. At 1 2 $\mathrm{h}$ after fibroblast-oocyte fusion, the resulted SCNT embryo was activated by ionomycin and 6-dimethylaminopurine, and human $K d m 4 d$ was injected into the SCNT embryos at $5 \sim 6 \mathrm{~h}$ after activation and treated with trichostatin A for $10 \mathrm{~h}$ following activation. For ICSI, a single sperm was injected into the cytoplasm of MII oocytes and the fertilization of oocytes was confirmed by the appearance of two pronuclei after about $6 \mathrm{~h}$ after injection. For trophoblast replacement, ICSI and SCNT were performed on the same day on same patch of collected oocytes in order to synchronize the development of two types of embryos.

\section{Trophoblast replacement}

First of all, a small slit was created on the polar side zona pellucida of a ICSI blastocyst by laser ablation. The ICSI-ICM was specifically hatched out of the zona pellucida through the slit after about $6 \mathrm{~h}$ of culturing, while the blastocoel remained expanded. About $5 \mathrm{~h}$ after the laser ablation on ICSI blastocysts, 
SCNT-ICM was obtained by immunosurgery. Then, the isolated SCNT-ICM was immediately injected into the ICSI blastocoel after another slit was created. Finally, the hatched endogenous ICSIIICM was removed by laser irradiation. Notably, 16 SCNT-ICMs were injected into $11 \mathrm{ICSI}$ blastocoels with the ratio of SCNTICM and ICSI trophoblast between $1 / 1$ and 3/1.

The immunosurgery was performed as described before with slight modification ${ }^{17}$. Briefly, the zona pellucida of the SCNT blastocyst was dissolved by pre-warmed acid Tyrode's solution and then washed by $\mathrm{H} 9$ medium for at least 3 times. The blastocysts were cultured in rabbit anti-monkey IgG whole serum (Bioss, bs-0335Rs) for 30 min, after 3 times washing in $\mathrm{H} 9$ medium they were transferred into Guinea pig complement serum (Fitzgerald, 32R-CP004) for another $30 \mathrm{~min}$. The intact SCNT-ICMs were isolated by gentle pipetting with a beveled pipette (around $50 \mu \mathrm{m}$ in diameter) in TH3 medium under micromanipulation. The SCNT-ICMs were injected into the ICSI blastocoels immediately isolating.

\section{Monkey embryo culture and transfer}

Monkey embryos were cultured in serum-free $\mathrm{H} 9$ medium at $37{ }^{\circ} \mathrm{C}$ in $5 \% \mathrm{CO}_{2}$ until 8-cell stage (day 3 after fertilization) and then were transferred into $\mathrm{H} 9$ containing $5 \%$ embryonic stem cell grade FBS. The medium was changed every two days until the blastocyst stage.

Within 2 hours of reconstruction, the reconstructed embryos were transferred into the oviducts of surrogates with fresh stigmas on the ovaries.

\section{STR analysis}

Ear tissue of the cloned rhesus monkey, donor fibroblasts and peripheral blood samples of the oocyte donor, sperm donor and surrogate mother were collected for short tandem repeat analysis. Locus-specific primers each containing a fluorescent dye (FAM/HEX/TMR) were used for PCR amplification in batches. FAM, HEX or TMR-labeled STR amplicons were diluted and mixed with internal size standard ROX500 and deionized formamide, followed by capillary electrophoresis on ABI PRISM 3730 genetic analyzer to obtain the raw data. Sequencer-generated raw data were analyzed with the program Gene Marker 2.2.0, which produces wave plots, Excel documents (including information such as size and genotype), and DNA profiles.

\section{mtDNA examination}

Total genomic DNAs were prepared from the ear tissue or blood samples of cloned monkey, surrogate mother, sperm donor, oocyte donor together with DNA from donor cells using the Genomic DNA extraction Kit according to the manufacturer's instructions (Tiangen, DP304-03). The whole mtDNA sequence was amplified by 13 pairs of primers to identify SNPs among the samples $(278 \mathrm{~F}$ : catgcagttgttgatcgcaccta, 278R: ggtttggcaagagtgggtt; 4061F: acaaccacaatcttcctaggcaca, 4061R: ggggaatgctggagattgcg; 4591F: cctgagaatccaaacttctccgtg, 4591R: agcatcctgatagtaggttgttgg; 5803F: ctgcaaacacctactctgcatcaa, 5803R: ggctcagggcagtgcctatga; 6040F: tcataggcactgccctgagcc, 6040R: gcggctaggactggtagagagag; 6988F: 
ataatcattgcaatccccaccggt, 6988R: gatggtgaaggatgggtcgttg; 8680F: acccctttctcaaccccaacaatc, 8680R: tggtaggaggtgggctagggag; 9543F: catagttaaacccagtccctggcc, 9543R: tcggagatggtgaagggtgcttc; 10051F: accacaggcttccacggac, 10051R: gggaggtttgttgtttgaatggct; 11530F: gagaccactctcattcctaccct, 11530R: tgttttctcgtgtgaagggggg; 13628F: cccttcctcacaggcttctactcc, 13628R: gttagtggtgtggttggttgtgtg; 15129F: ggactccaaccataactaacggca, 15129R: gctgtcaatggcgtatcctcctc; 16038F: cactatcggccaagtagcatccat; 16039R: gcatccgtggtgaggaggattat). DNA was amplified with 35 cycles of $95^{\circ} \mathrm{C}$ for $30 \mathrm{~s}, 57^{\circ} \mathrm{C}$ for $30 \mathrm{~s}$, and $72{ }^{\circ} \mathrm{C}$ for $1 \sim 1.5 \mathrm{~min}$, followed by a 5 -min extension at $72^{\circ} \mathrm{C}$. The PCR products were used for sequencing and the results were used for the SNP analysis.

\section{In vitro transcription of $K d m 4 d$}

For $K d m 4 d$ in vitro transcription, CDS of human $K d m 4 d$ gene was cloned from the cDNA plasmid provided by Dr. J.H. Han of Xiamen University. The DNA template was amplified by the T7 promoter containing primer $(\mathrm{F}$ :

TTAATACGACTCACTATAGGGATGGAAACTATGAAGTCTAAGGCCAACT, R: ATATAAA GACAGCCCGTGGACTTAGG). T7-Kdm4dPCR product was purified and used as the template for in vitro transcription using mMESSAGE mMACHINE T7 ULTRA kit (Life Technologies, AM1345). The RNA products were purified using MEGA clear kit (Life Technologies, AM1908) and eluted in RNase-free water.

\section{Alizarin red staining}

Whole placentas were fixed in $4 \%$ paraformaldehyde for 1 month before they were sectioned. Since the placentas are usually extraordinary large and can hardly be intact sectioned, the placentas were diced and the tissues were imbedded into pre-melted paraffin. After three times of paraffin bathing, the tissues were put into paraffin block molds. After cooling, the tissues were sectioned into $5 \mu \mathrm{m}$ thick slices and float them on clean water at room temperature. Pick the slices up to glass slides gently and dried for 1 2 h. Then, put them into a $60^{\circ} \mathrm{C}$ drying oven for $30 \mathrm{~min}$ to melt the wax. The resulting slides were dewaxed in xylene and decreased concentration of ethanol $(100 \%, 90 \%, 80 \%$ and $70 \%)$ and the slides were ready for AR staining after water flushing gently.

Alizarin red staining was performed as the manufacture's instruction (Servicebio, G1038). Briefly, the slides were incubated in alizarin red solution for 5 minutes followed by three times of washing. After drying for $4 \mathrm{~h}$ in $60^{\circ} \mathrm{C}$, the slides were incubated in xylene for clearing and finally mount with synthetic resin.

\section{Morphometry of placenta calcification}

The total calcified area and total area of all slides of each placenta was calculated by Image $J$ and the calcification index (CCl) was calculated as:

$\mathrm{CCl}=$ Total calcified area of all slides of each placenta/Total area of all slides of each placenta $* 100 \%$. 
The average thickness of each placenta was measured by ImageJ. Briefly, the thickness of each slide was measured and the average thickness of each placenta was calculated as:

Thickness $=$ Thickness of all slides of each placenta/Total number of slides of each placenta .

\section{References}

1. Kato, Y. et al. Eight Calves Cloned from Somatic Cell of a Single Adult. Science 282, 2095-2098 (1998).

2. Polejaeva, I.A. et al. Cloned pigs produced by nuclear transfer from adult somatic cells. Nature 407 , 86-90 (2000).

3. Baguisi, A. et al. Production of goats by somatic cell nuclear transfer. Nat Biotechnol 17, 456-461 (1999).

4. Chesné, P. et al. Cloned rabbits produced by nuclear transfer from adult somatic cells. Nature Biotechnology 20, 366-369 (2002).

5. Lee, B.C. et al. Dogs cloned from adult somatic cells. Nature 436, 641 (2005).

6. Liu, Z. et al. Cloning of Macaque Monkeys by Somatic Cell Nuclear Transfer. Cell 172, 881-887 e887 (2018).

7. Liu, Z. et al. Cloning of a gene-edited macaque monkey by somatic cell nuclear transfer. National Science Review 6, 101-108 (2019).

8. Sparman, M.L., Tachibana, M. \& Mitalipov, S.M. Cloning of non-human primates: the road "less traveled by". Int J Dev Biol 54, 1671-1678 (2010).

9. Meng, L., Ely, J.J., Stouffer, R.L. \& P.Wolf, D. Rhesus Monkeys Produced by Nuclear Transfer. Biology of Reproduction 57, 454-459 (1997).

10. Chang, S.W. \& Platt, M.L. Oxytocin and social cognition in rhesus macaques: implications for understanding and treating human psychopathology. Brain Res 1580, 57-68 (2014).

11. Banks, M.L. \& Negus, S.S. Insights from Preclinical Choice Models on Treating Drug Addiction. Trends Pharmacol Sci 38, 181-194 (2017).

12. Lin, J. et al. Defects in trophoblast cell lineage account for the impaired in vivo development of cloned embryos generated by somatic nuclear transfer. Cell Stem Cell 8, 371-375 (2011).

13. Chavatte-Palmer, P. et al. Review: Placental perturbations induce the developmental abnormalities often observed in bovine somatic cell nuclear transfer. Placenta 33 Suppl, S99-S104 (2012).

14. Ao, Z. et al. Birth weight, umbilical and placental traits in relation to neonatal loss in cloned pigs. Placenta 57, 94-101 (2017).

15. Zheng, P. Effects of in vitro maturation of monkey oocytes on their developmental capacity. Anim Reprod Sci 98, 56-71 (2007).

16. Zheng, P. et al. 17Beta-estradiol and progesterone improve in-vitro cytoplasmic maturation of oocytes from unstimulated prepubertal and adult rhesus monkeys. Hum Reprod 18, 2137-2144 (2003). 
17. Tachibana, M. et al. Generation of chimeric rhesus monkeys. Cell 148, 285-295 (2012).

\section{Table 1}

Table 1 is available in the supplementary files section.

\section{Figures}




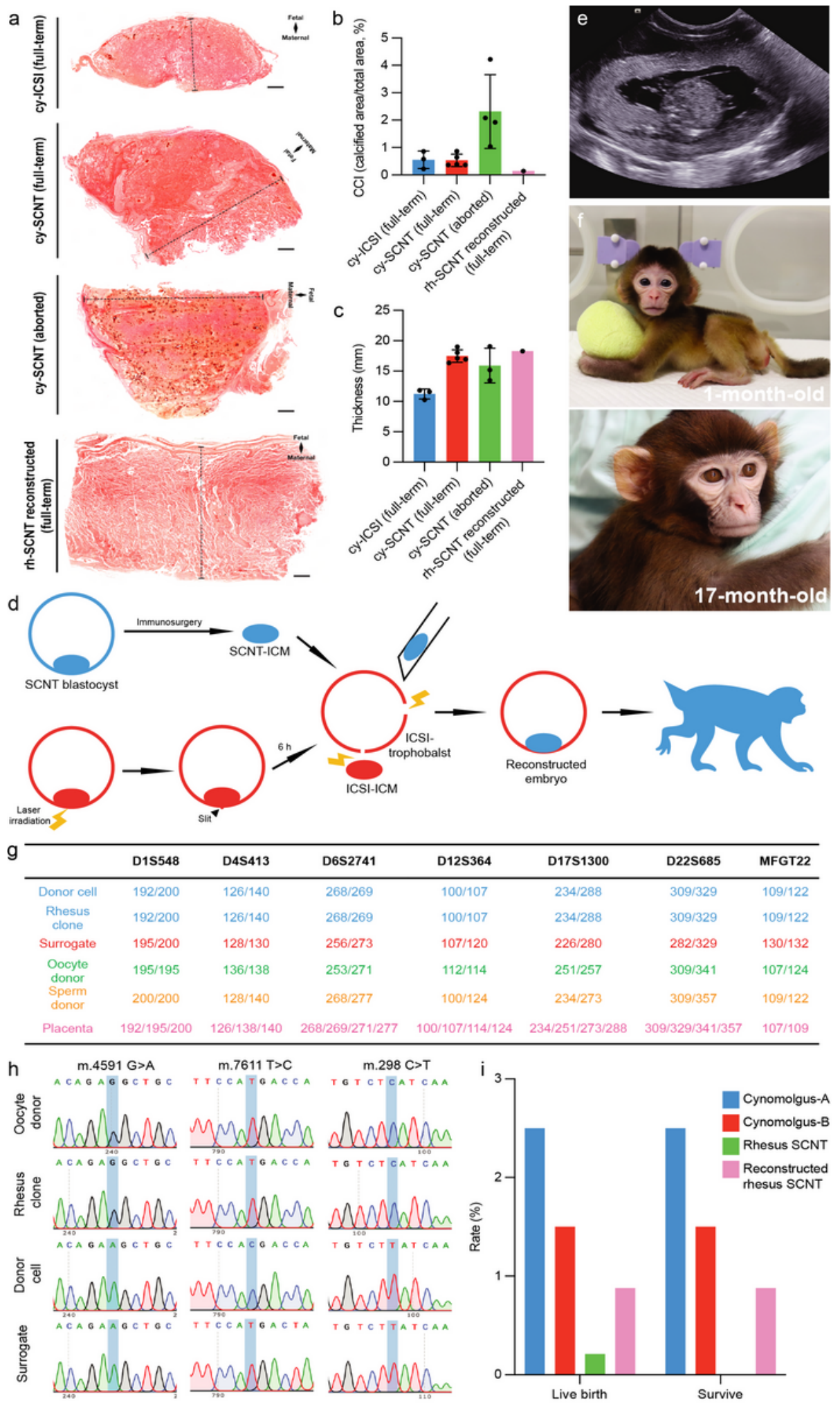

Figure 1

Trophoblast replacement led to healthy live birth of SCNT hesus monkey. (a) Representative images of AR staining showing the thickness and calcification of full-term ICSI and SCNT placentas, late stage aborted SCNT placentas, and the reconstructed SCNT placenta. Scale bar, $2 \mathrm{~mm}$. The maternal and fetal surface were indicated as in the figure. (b) The comparison of calcification index (CCl) of full-term ICSI $(n=3)$ and SCNT $(n=5)$ placentas, late stage aborted SCNT $(n=4)$ placentas, and the full-term 
reconstructed SCNT placenta $(n=1)$. (c) The comparison of the thickness of full-term ICSI $(n=3)$ and SCNT $(n=5)$ placentas, late stage aborted SCNT $(n=3)$ placentas, and the full-term reconstructed SCNT placenta $(n=1)$. The average placenta thickness was calculated by the length between the maternal and fetus layer of each AR slide per placenta. (d) Schematic illustration of the trophoblast replacement technology. (e) Ultrasound examination of the reconstructed rhesus SCNT fetus on gestation day 60. (f) The cloned rhesus monkey derived by trophoblast replacement at 1-month and 17-month. (g) STR analysis confirmed the genomic origin of the cloned monkey. (h) Representative alleles showing the mtDNA origin of the cloned rhesus monkey. (i) Trophoblast replacement improves the rates of full-term live birth and survival of rhesus monkey SCNT embryos.

\section{Supplementary Files}

This is a list of supplementary files associated with this preprint. Click to download.

- reprogrammingSupplementaryMaterials.docx

- Movie1.mp4

- nreditorialpolicychecklist.pdf

- table1.jpg

- ReportingSummary.pdf 